\title{
Experimental Determination of Thermal Conductivity and Diffusivity of New Building Insulating Materials
}

\author{
Ines Boulaoued *, Ines Amara and Abdallah Mhimid \\ Thermal System Energetic Laboratory Research (LESTE), National School Engineering of \\ Monastir. Avenue Ibn Jazzar. 5019 Tunisia \\ Email: ines_boulaoued_01@yahoo.fr
}

\begin{abstract}
The present study is performed within the framework of the program of thermal and energy regulation of new buildings in Tunisia. This work presented an experimental determination of the thermo physical properties of insulating materials used in building. For this purpose, a new experimental apparatus was constructed according to the Tunisian standards. An analytical method is used in determining the thermal conductivity, and two analytical models are used to determine the thermal diffusivity. The obtained values of various materials were measured. They are acceptable compared with the values given by the available published results. Original insulating materials using palm tree fibers and seaweed fibers in a cement matrix were studied in this work.
\end{abstract}

Keywords: Seaweed fibers, Palm tree fibers insulation, Conservation of energy, Thermal conductivity, Thermal diffusivity.

\section{INTRODUCTION}

Due to the increasing population energy consumption in the sector of building, and the hard economic situation, it is necessary to control energy during the building of buildings. The use of thermal insulation is one of the most important energy conservation in building. The majority of thermal insulations frequently used for building insulation are chosen for their ability to block heat flux.

Despite the increased use of insulation materials, detailed and systematic studies of their thermal characteristics are limited. In this perspective, we are interested in determining the thermo physical properties of materials used in building. It is of primary interest to determine the thermal conductivity and thermal diffusivity of different insulators used in building.

There are several methods for measuring thermal conductivity. Each method is suitable for a limited range of materials, depending on the thermal properties and the temperature. Jose Luis Vivancos [1] developed a fast and reliable protocol to determine the characteristics of building materials. It is of importance to develop environment friendly houses with an efficient energy design. M. Sait Soylemez [2] shows that the value of the effective thermal conductivity of building bricks is important for engineering applications. The thermal conductivity of thermal insulation is usually measured using steady-state techniques such as the Box method El Bakkouri [3], L. Mr Voumbo and al [4], H. Ezbakhe [5], Degiovanni (1994) [6] and I. Boulaoued et al [7]. The hot wire method (THW) for the measurement of the thermal conductivity of composites, proposed by Carslaw et al [8], and used by several authors like $\mathbf{M}$ Lachheb et al [9]. This method, is a transient technique based on the measurement of the temperature rise of a linear heat source embedded between two tested materials.

The method of the guarded hot plate used by several authors like W. Hemminger et al [10], F. Deponte et al [11]. B. Martin et al [12]. Such methods require large samples and long measurement times. Generally, these methods of measurement present some difficulties such as the existence of resistance of contact and the axial and radial lost flow.

In order to create a reliable thermal design system, it is essential to characterize the materials and their basic properties accurately. Gi-Won Nam [13] et al used the cyclic heating method to measure the thermal diffusivity for a high-temperature, porous material as an insulating material for a spacecraft Kuk-Hee [14] Lim assumed that the flash method is the most popular method of measuring the thermal diffusivity of solids. The first approach was proposed in 1961 by Parker et al [15], B. Hay. [16], L. Vozar [17] and Degiovanni [18-19]. It deals with the estimation of the in-depth diffusivity of a sample. It consists in applying a very short and uniform burst of energy at the front face of a sample in order to generate one dimensional heat transfer. The resulting temperature which rises at the back face of the sample is measured. The model corresponding to the experiment shows that the in-depth 
diffusivity can be computed once the half-rise time $t_{1 / 2}$ is estimated.

The present paper has two main objectives. The first objective is the measurement of thermal properties of some commonly used insulating materials produced by local manufacturers in Tunisia. The second is to present the design of a new apparatus, fast and accurate for measuring the thermal conductivity and diffusivity of insulating materials.

\section{EXPRIMENTAL}

The experimental apparatus [20] is schematically shown in figure 1. It consists of a plexiglas box with dimensions of $0.50 \times 0.30 \times 0.30 \mathrm{~m}^{3}$ and a mobile cover. It is highly insulated thanks to a thick layer of expanded polystyrene in order to reduce the eventual heat loss between the outer plate and room temperature.

It contains a heat exchanger circulating hot water thanks to a pump with an electric heater. This heat exchange allows the measurement of the thermal diffusivity of the sample placed between heat exchange and the insulating material. The samples must to have a square section of $27 \mathrm{~cm}$ sides and variable thicknesses. Heat exchanger formed by a copper box has dimensions of $0.27 \times 0.27 \mathrm{~m}^{2}$. The temperatures are measured with platinum probes. Each sample is provided with two surface probes fixed on the respective faces. All temperature sensors are connected to an AGILENT 34970A data acquisition system allowing the acquisition and the storage of temperature signals on a personal computer with an accuracy of $0.1{ }^{\circ} \mathrm{C}$ (see Figure1).

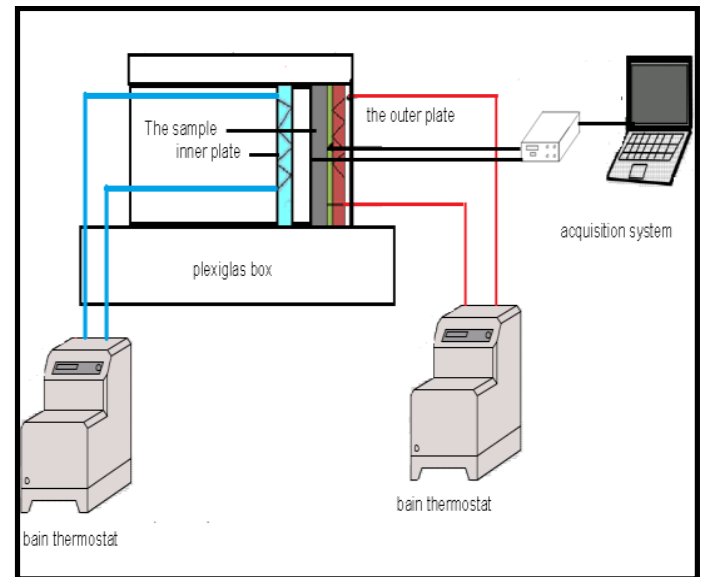

Figure 1. Experimental apparatus

\section{MATHEMATICAL MODELING}

\subsection{Thermal conductivity}

In steady one-dimensional conditions, one can determine the thermal conductivity by measuring the heat flux through the sample and the temperature difference between its two faces. In order to insure steady-state a condition, the heat exchanger is provided with five surface probes (platinum probes) fixed on various places (see Figure 2). Figure 3 shows that the measurements were carried out after at least 30 min from setting the experimental conditions

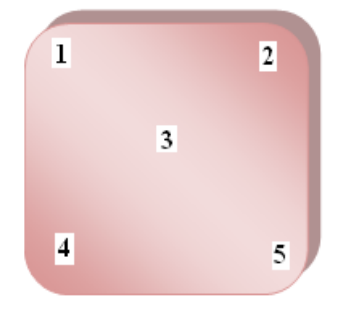

Figure 2. Location of the probes on the face of the heat exchanger

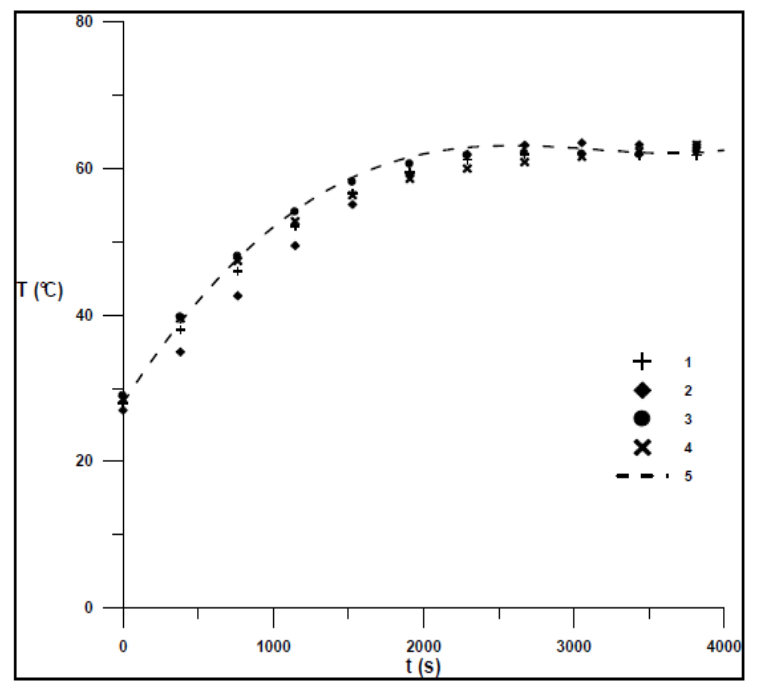

Figure 3. The steady state conditions

The equation of the thermal balance is written as follow:

$\phi_{p}+\phi_{r}=\phi_{e c}$

The heat transmitted by conduction $\phi_{\mathrm{ec}}$ through the sample is written as

$\phi_{e c}=\frac{\lambda S}{e}\left(T_{c}-T_{f}\right)$

where $\mathrm{S}$ is the sample effective heat exchange area, e is the thickness, $T_{f}$ and $T_{c}$ are respectively the temperatures of the cold and hot faces.

The heat received by the ambient water is given by the flowing equation:

$$
\phi_{r}=\dot{m} \cdot \operatorname{Cp} \cdot\left(T_{s e}-T_{e e}\right)
$$

where $\mathrm{m}$ is the mass flow, $C p$ is the specific heat, $T_{s e}$ and $T_{e e}$ are respectively the hot and cold water temperatures.

The heat loss from the surface $\phi_{P}$ was obtained experimentally in the qualification tests of the apparatus, with a reference sample whose thermal conductivity is known (see Table 1). 
Table 1. Measured values of $\phi_{\mathrm{p}}$

\begin{tabular}{lcc}
\hline Samples & $\lambda$ & $\phi_{p}$ \\
\hline 1 & 0.039 & 0.822 \\
2 & 0.033 & 0.818 \\
3 & 0.038 & 0.816 \\
4 & 0.036 & 0.810 \\
5 & 0.040 & 0.821 \\
\hline
\end{tabular}

Using the following expression:

$\phi_{p}=\frac{\lambda S}{e}\left(T_{c}-T_{f}\right)-\dot{m} \cdot C p \cdot\left(T_{s e}-T_{e e}\right)$

It was found in a previous study, and confirmed here, that $\phi_{p}=0.815 \mathrm{~W}$

The expression of thermal conductivity of the sample is obtained by combining the above relations:

$\lambda=\left[\dot{m} \cdot C p \cdot\left(T_{s e}-T_{e e}\right)+\phi_{p}\right] \frac{e}{S\left(T_{c}-T_{f}\right)}$

This expression is valid for steady state conditions. For the apparatus configuration and test conditions of the present study, the maximum measurement error was estimated to $8 \%$ for the thermal conductivity.

\subsection{Thermal diffusivity}

The principle of this method can be described as follows. The sample receives a thermal impulse. The resulting heat flux crosses the sample from the irradiated face towards the opposite face is adiabatic. Thermocouples fixed in the middle of the sample faces and connected to a computer data acquisition card yield temperature thermograms (timeseries) for the respective faces of the sample. These thermograms can be used to calculate the thermal diffusivity [20].

During this work we supposed the following conditions:

-The one-dimensional unsteady conduction

-The incident energy of the impulse must be distributed uniformly on the excited face.

-The studied medium could be considered homogeneous.

In this case, the one-dimensional unsteady conduction is represented by the equation of Fourier:

$\frac{\partial^{2} T}{\partial x^{2}}=\frac{1}{\alpha} \frac{\partial T}{\partial t}$

Initially the temperature is uniform in all the medium

$T(x, 0)=T_{i}$

The boundary conditions are:

The interior side was heated at the temperature

$T(0, t)=T_{0}$
The exterior side was isolated by the wool of rock outside we can write

$-\left.\lambda \frac{\partial T(x, t)}{\partial x}\right|_{x=e}=h\left(T-T_{i}\right)$

where $\lambda$ is the thermal conductivity, $h$ is the convective coefficient exchange and $T_{i}$ is the initial face temperature.

3.2.1 Analytical model: Separation of variables [20]

The solution of equation (7) may be obtained by introducing a new variable $\theta$ expressed by:

$\theta(x, t)=\frac{T(x, t)-T_{0}}{T_{i}-T_{0}}$

When the convective coefficient exchange is assumed $\mathrm{h}=0$, the final solution of the temperature is expressed as:

$$
\frac{T(x, t)-T_{0}}{T_{i}-T_{0}}=\frac{4}{\pi} \sin \left(\frac{\pi}{2 e} x\right) \exp \left\{-\alpha t\left[\frac{\pi}{2 e}\right]^{2}\right\}
$$

where $\alpha$ is the thermal diffusivity, e is the sample thickness, $\mathrm{T}_{\mathrm{i}}$ and $\mathrm{T}_{0}$ are the initial and exterior face temperature.

3.2.2 Analytical model: Laplace method

The solution may be obtained by introducing a new variable expressed by:

$T^{*}(x, t)=T(x, t)-T_{i}$

Initially and the boundary conditions are given as:

$\mathrm{T}^{*}(0, \mathrm{t})=\mathrm{T}_{0}-\mathrm{T}_{\mathrm{i}}$

$\mathrm{T} *(\mathrm{x}, 0)=0$

Using the time $t_{1 / 2}$ obtained for the experimental curve and the flowing expression we can determine the value of thermal diffusivity

$$
\frac{1}{2} \frac{T_{\infty}-T_{i}}{\left(T_{0}-T_{i}\right)}=\operatorname{erfc}\left(\frac{e}{2 \sqrt{\alpha t_{1 / 2}}}\right)
$$

\section{RESULTATS AND DISCUSSION}

Measurements of thermal conductivity and thermal diffusivity were conducted for a total of five commercially insulating materials and new insulators. Two new insulators were studied in this paper. First is composed of short palm tree fibers in a cement matrix. With 5 mass percentages of palm fibers were considered; namely, 5, 20, 30, 40, and $60 \%$. The second is composed of seaweed fibers in a cement matrix With 5 mass percentages of seaweed fibers were considered; namely, 5, 10, 20, 30, and $40 \%$. 


\subsection{Thermal conductivity}

Thermal conductivities of commercially insulating materials, which were obtained by the new apparatus according to Eq. (5), are presented in Table 2. The measured values of these materials were in satisfactory agreement within $8 \%$ with typical values given by the box method.

Table 2. Measured values of the thermal conductivity of commercially available insulating materials.

\begin{tabular}{lllll}
\hline \multicolumn{5}{c}{ Thermal conductivity $\lambda\left(\mathbf{W} \cdot \mathbf{~ m}^{-1} \mathbf{K}^{-1}\right)$} \\
\hline \multirow{2}{*}{ Samples } & $\begin{array}{l}\text { New } \\
\text { method }\end{array}$ & $\begin{array}{l}\text { Box } \\
\text { method }\end{array}$ & $\begin{array}{l}\text { Relatif } \\
\text { difference }(\%)\end{array}$ & BIB \\
\hline 5 & 0.0408 & 0.04 & 1.96 & $0.035-0.040$ \\
6 & 0.034 & 0.032 & 5.88 & $0.029-0.036$ \\
7 & 0.043 & 0.041 & 4.65 & $0.038-0.065$ \\
8 & 0.0397 & 0.040 & 0.75 & $0.038-0.065$ \\
9 & 0.0421 & 0.045 & 6.88 & $0.038-0.065$ \\
\hline
\end{tabular}

The results of the effective thermal conductivity of the palm tree fiber content materials are presented in Table 3 [Fig. 4].

Table 3. Measured values of the thermal conductivity of the palm tree based materials for different fiber contents.

\begin{tabular}{llll}
\hline & \multicolumn{2}{l}{ Thermal conductivity $\lambda\left(\mathbf{W} \cdot \mathbf{~ m}^{-\mathbf{1}} \mathbf{K}^{-\mathbf{1}}\right)$} \\
\hline $\begin{array}{l}\text { Palm Fiber } \\
\text { mass ratio (\%) }\end{array}$ & New method & Box method & $\begin{array}{l}\text { Relatif } \\
\text { difference }\end{array}$ \\
\hline 60 & 0.035 & 0.033 & 5.714 \\
40 & 0.037 & 0.036 & 2.703 \\
30 & 0.048 & 0.046 & 4.167 \\
20 & 0.055 & 0.056 & 1.82 \\
5 & 0.094 & 0.091 & 3.191 \\
\hline
\end{tabular}

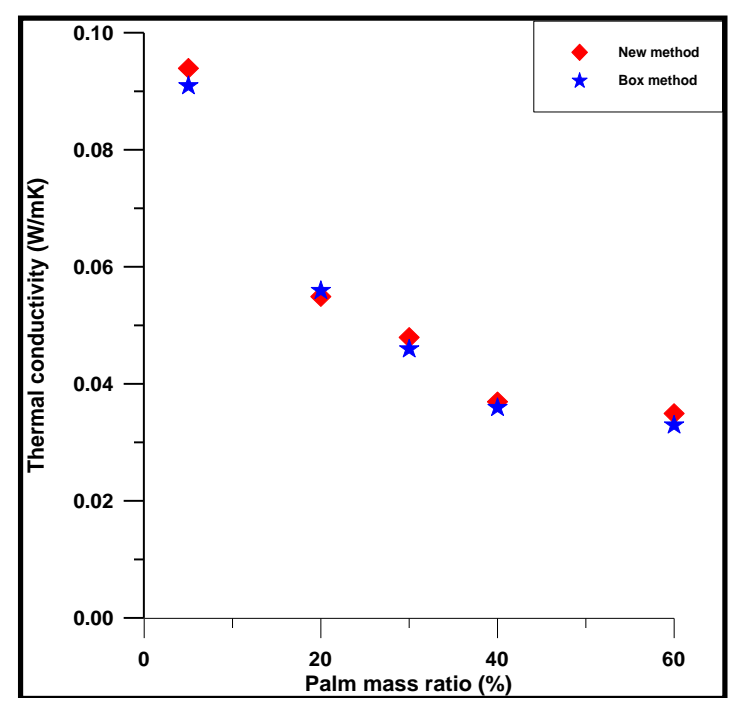

Figure 4. Variation of the thermal conductivity as a function of the palm mass ratio
The effective thermal conductivity decreased drastically with increasing fiber content and then its values leveled off at around a mass ratio of $40 \%$.

The results for the effective thermal conductivity of the Seaweed fiber content materials are presented in Table 4 and [Fig. 5].

Table 4. Measured values of the thermal conductivity of the Seaweed based materials for different fiber contents.

\begin{tabular}{llll}
\hline & \multicolumn{3}{l}{ Thermal conductivity $\boldsymbol{\lambda}\left(\mathbf{W} . \mathbf{~ m}^{-1} \mathbf{K}^{-1}\right)$} \\
\hline $\begin{array}{l}\text { Seaweed Fiber } \\
\text { mass ratio (\%) }\end{array}$ & New method & $\begin{array}{l}\text { Box } \\
\text { method }\end{array}$ & $\begin{array}{l}\text { Relatif } \\
\text { difference }\end{array}$ \\
\hline 40 & 0.049 & 0.047 & 4,08 \\
30 & 0.057 & 0.054 & 5,26 \\
20 & 0.069 & 0.066 & 4,34 \\
10 & 0.075 & 0.074 & 1,33 \\
5 & 0.089 & 0.088 & 1,12 \\
\hline
\end{tabular}

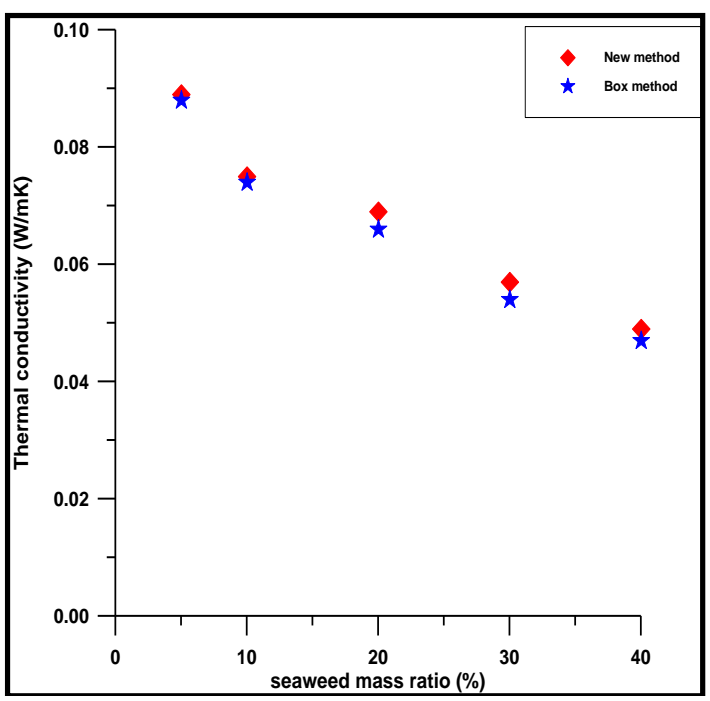

Figure 5. Variation of the thermal conductivity and as a function of the seaweed mass ratio

The effective thermal conductivity decreased with increasing fiber content. Materials frequently used for building insulation are chosen for their low thermal conductivity and their ability to block heat flux. In fact, for mass ratios higher than $20 \%$ the obtained material is competitive in terms of thermal conductivity. Moreover, it has the advantage of being a natural, harmless and lessexpensive product.

\subsection{Thermal diffusivity}

The measured values of thermal diffusivity for the commercially available samples and the new insulators are summarized in Tables 5 . 
Table 5. Measured values of the thermal diffusivity of commercially available insulating materials.

\begin{tabular}{lllll}
\hline Samples & \multicolumn{4}{l}{ Thermal diffusivity $\boldsymbol{\alpha} \times \mathbf{1 0}^{-7}\left(\mathbf{m}^{2} \mathbf{~ s}^{-1}\right)$} \\
\hline & $\begin{array}{l}\text { Box } \\
\text { method }\end{array}$ & $\begin{array}{l}\text { Laplace } \\
\text { method }\end{array}$ & $\begin{array}{l}\text { Separation of } \\
\text { variables method }\end{array}$ & BIB \\
\hline 5 & 3.58 & 3.42 & 3.18 & 3.64 \\
6 & 4.98 & 4.78 & 4.58 & 5.25 \\
7 & 3.53 & 3.71 & 3.05 & 3.64 \\
8 & 3.22 & 3.141 & 3.01 & 3.64 \\
9 & 3.30 & 3.3115 & 3.03 & 3.64 \\
\hline
\end{tabular}

The thermal diffusivity was calculated according to the method of Separation of variables, using Eq. (10), and according to the Laplace method, using Eq. (13). The obtained values of the two models conformed and agreed well with the flash method.

For the palm tree-based insulators, we found that an increase in the fiber mass ratio led to a decrease in the effective thermal diffusivity [Table 6 and Fig.6].

Table 6. Measured values of the thermal diffusivity of the palm tree based materials for different fiber contents

\begin{tabular}{llll}
\hline & \multicolumn{2}{l}{ Thermal diffusivity $\alpha \times \mathbf{1 0}^{-7}\left(\mathbf{m}^{2} \mathbf{s}^{-1}\right)$} \\
\hline $\begin{array}{l}\text { Palm mass } \\
\text { ratio (\%) }\end{array}$ & $\begin{array}{l}\text { Flash } \\
\text { method }\end{array}$ & $\begin{array}{l}\text { Laplace } \\
\text { method }\end{array}$ & $\begin{array}{l}\text { Separation of } \\
\text { variables } \\
\text { method }\end{array}$ \\
\hline 60 & 1.184 & 1.222 & 1.151 \\
40 & 1.408 & 1.452 & 1.361 \\
30 & 1.601 & 1.558 & 1.497 \\
20 & 1.696 & 1.660 & 1.56 \\
5 & 1.988 & 2.087 & 1.89 \\
\hline
\end{tabular}

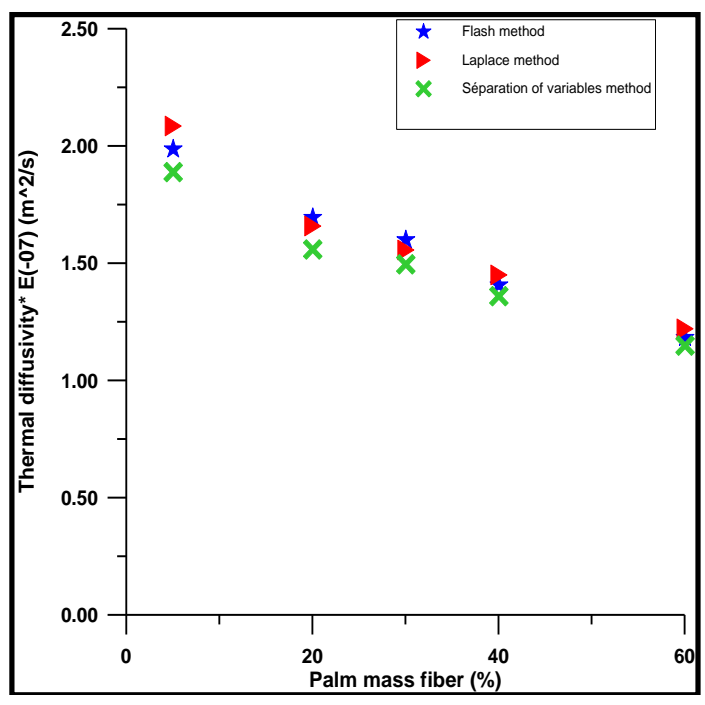

Figure 6. Variation thermal diffusivity as a function of the palm mass ratio

In addition, the effective thermal diffusivity became less sensitive to the value of this ratio for intervals corresponding to high percentages. So, increasing the mass ratio of the fiber is favorable in terms of thermal inertia. Combining the results for thermal conductivity and diffusivity one can deduce that the more fiber content, the better is the insulating material. The limit on this fiber content would be set by the mechanical performance of the material, as the fiber content weakens the integrity of the material.

Table 7 and Fig. 7 present the experimental values of the thermal diffusivity for the seaweed samples as a function of the fiber content. We can see that the thermal diffusivity decreases with the mass ratio of the fibers, with an abrupt decrease when the latter exceeds $40 \%$.

Table 7. Measured values of the thermal thermal diffusivity of the Seaweed based materials for different fiber contents.

\begin{tabular}{cccc}
\hline & \multicolumn{3}{c}{ Thermal diffusivity $\boldsymbol{\alpha} \times \mathbf{1 0}^{-\mathbf{7}}\left(\mathbf{m}^{\mathbf{2}} \mathbf{s}^{-\mathbf{1}}\right)$} \\
\hline $\begin{array}{c}\text { Seaweed mass } \\
\text { ratio }(\%)\end{array}$ & $\begin{array}{l}\text { Flash } \\
\text { method }\end{array}$ & $\begin{array}{l}\text { Laplace } \\
\text { method }\end{array}$ & $\begin{array}{c}\text { Separation of } \\
\text { variables method }\end{array}$ \\
\hline 40 & 1.113 & 1.141 & 1.07 \\
30 & 1.166 & 1.193 & 1.12 \\
20 & 1.219 & 1.240 & 1.131 \\
10 & 1.30 & 1.343 & 1.270 \\
5 & 1.532 & 1.572 & 1.463 \\
\hline
\end{tabular}

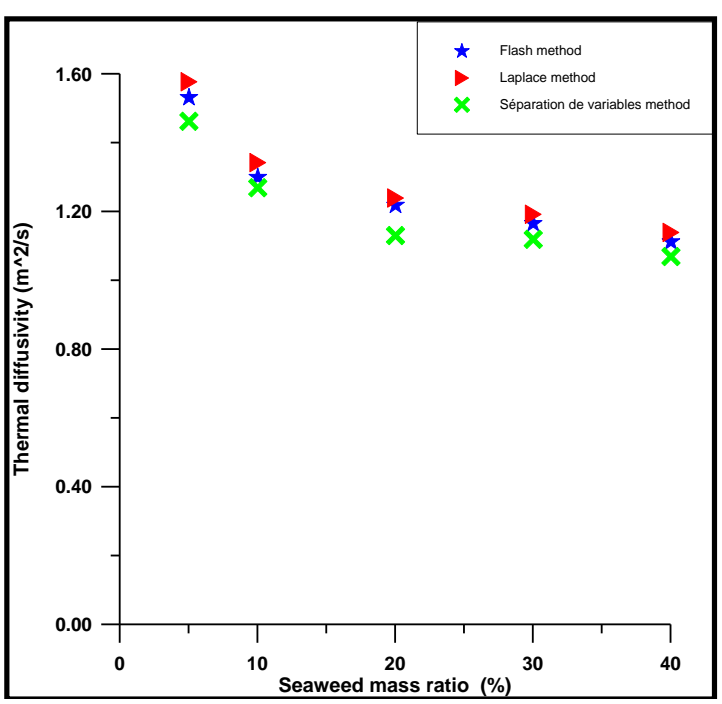

Figure 7. Variation of thermal diffusivity as a function of the seaweed mass ratio

We found that an increase in the fiber mass ratio led to a decrease in the effective thermal diffusivity. In addition, the effective thermal diffusivity became less sensitive to the value of this ratio for intervals corresponding to high percentages. Combining the results for thermal conductivity and diffusivity, one can deduce that the more the fiber content is the better the insulating material becoms. The limit on this fiber content would be set by the mechanical performance of the material, as the fiber content weakens integrity the material. One can deduce that the obtained materials were competitive in terms of thermal conductivity and had the advantage of being a natural, harmless and less expensive product.

A comparison with available flash method results also is presented. It shows that the measured values obtained by the two models belong to the expected intervals. 


\section{CONCLUSION}

The present study is performed within the framework of the program of thermal and energy regulation of new buildings in Tunisia. This work presented an experimental determination of the thermo physical properties. In this article, a new experimental apparatus was constructed according to the Tunisian standards. An analytical method is used to determine the thermal conductivity. The measured values of the commercially insulating materials obtained by the new apparatus were in satisfactory agreement, within $6 \%$, with typical values given by the box method.

Two analytical models are used to determine the thermal diffusivity. The results suggest that the measured values of various materials obtained are acceptable compared with the values given by the flash method.

The original insulating materials using palm tree fibers and seaweed in a cement matrix present properties that are a function of the fiber content mass ratios and have values that are generally comparable to synthetic insulating materials. We can note that the thermal conductivity and diffusivity of these insulators decreased rapidly with the ratio of fiber mass.

\section{ACKNOWLEDGMENT}

The financial support of the Tunisian National Agency for the Management of Energy (ANME) is gratefully acknowledged.

\section{REFERENCES}

[1] J. L. Vivancos, J. Soto, I. Perez, Jose V. Ros-Lis and R. Martınez-Manez, "A new model based on experimental results for the thermal characterization of Bricks," Building and Environment, 44, pp. 10471052, 2009. DOI: 10.1016/j.buildenv.2008.07.016.

[2] M. Sait Soylemez, "On the effective thermal conductivity of building bricks," Building and Environment, 34, pp. 1-5, 1999. DOI: 10.1016/S0360-1323(98)00002-X.

[3] A. El Bakkouri, S. Boussaid, H. Ezbakhe, T. Ajzoul and A. Elbouardi, «Méthode générale d'identification paramétrique: Application à l'étude thermophysique de quelques matériaux locaux utilisés dans la construction au Maroc »(Plâtre - Liège - Brique creuse) Revue canadienne de Génie Civil, vol. 27, no. 4, pp. 628-631, 2000.

[4] L. M. Voumbo, B. Dieng, S. Tamba, S. Gaye, M. Adj and G. Sissoko, «Automatisation de la mesure de la conductivité thermique et de la diffusivité thermique par la méthode de boites, »Sci., vol. 7, pp 82 - 95, 2007.

[5] H. Ezbakhe, « Caractérisation thermiques et mécaniques des matériaux poreux utilisés comme isolants simples ou porteurs, » Thèse de doctorat, UCPB Lyon 1, 1986.

[6] A. Degiovanni, « Conductivité et diffusivité thermique des solides, » Technique de l'Ingénieur traité Mesures et Contrôle, R 2850, 1994.

[7] I. Boulaoued, M. S. Guellouz, A. Mhimid and A. Jemni, "Expermental determination of the thermo- physical properties of building insulating materials," Special Topics \& Reviews in Porous Media - An International Journal, vol. 3, no. 2, pp. 97-108, 2012.

[8] R. Yezou, «Contribution à l'étude des proprieties thermophysiques des matériaux de construction cohérents et non cohérents, » Thèse de Doteur Ingénieur, INSA, Lyon, France, 1978.

[9] Thermicar, « Contrôle non destructif et caractérisation thermique, »Fil chaud-Plan chaud. Thermoconcept, 16-17 Mars, 2005.

[10] W. Hemminger and R. Jugel. A "Guarded hot-plate apparatus for thermal conductivity measurements over the temperature range -75 to $200^{\circ} \mathrm{C}$," International Journal of Thermophysics, vol. 6, no. 5. 1985.

[11] F. Deponte and P. Phillipo, "Design criteria for the hot plate appartus. Heat transmission measurement in thermal insulations," ASTM STP 544, American Society for Testing and Materials, pp. 97-117, 1997.

[12] B. Martin, J. F. Durastanti and B. Peureux, « Méthode d'identification de la conductivité thermique des matériaux isolants, »Entropie, vol. 147, pp. 47-52, 1994.

[13] Gi-Won Nam, Cheol-Won Kong, Yeong-Moo Yi and Akira Ohnishi, "Thermal diffusivity measurement of BMS 10-102 thermal insulation material in a vacuum condition using a cyclic heating method," Thermochimica Acta, vol. 494, pp. 123-128, 2009.

[14] Kuk-Hee Lim, Seog-Kwang Kim and Myung-Kyoon Chung, "Improvement of the thermal diffusivity measurement of thin samples by the flash method," Thermochimica Acta, vol. 494, pp. 71-79, 2009. DOI: $10.1016 /$ j.tca.2009.04.019.

[15] Parker W. J., Jenkins R. J., Butler C. P. and Abbot G. L., "Flash Method of determining thermal diffusivity. heat capacity and thermal conductivity," J. of Appl. Phys., vol. 32, no. 9, pp. 1679-1684, 1961. DOI: 10.1063/1.1728417.

[16] B. Hay, J. R. Filtz, J. Hameury and L. Rongione, "Uncertainty of thermal diffusivity measurements by laser flash method," Int. J. Thermophys, vol. 26, no. 6, pp. 1883-1898, 2005. DOI: $10.1007 / \mathrm{s} 10765-005-$ 8603-6.

[17] L. Vozar and W. Hohenauer, "Uncertainty of thermal diffusivity measurements using the laser flash method," Int. J. Thermophys, vol. 26, no. 6, pp. 1899-1915, 2005. DOI: 10.1007/s10765-005-8604-5.

[18] A. Degiovanni, « Contribution à l'étude de la diffusivité thermique, » Thèse de Doctorat d'état, Lyon, France, 1975.

[19] A. Degiovanni, « Identification de la diffusivité thermique par l'utilisation des moments temporels partiels, » High Temperature - High Pressure, vol. 17. pp. 683-689, 1985.

[20] W. K. Chen, Linear Networks and Systems. CA: Wadsworth: Belmont, 1993, pp. 123-135.

\section{NOMENCLATURE}

$\begin{array}{ll}\mathrm{CP} & \text { specific heat, } \mathrm{J} \cdot \mathrm{kg}^{-1} \cdot \mathrm{K}^{-1} \\ \mathrm{e} & \text { thickness, } \mathrm{m} . \\ \mathrm{S} & \text { Sample area, } \mathrm{m}^{2} \\ \mathrm{~T} & \text { Temperature, } \mathrm{K}\end{array}$




\section{Greek symbols}

$\alpha \quad$ thermal diffusivity, $\mathrm{m}^{2} . \mathrm{s}^{-1}$

$\phi \quad$ heat transmitted, $\mathrm{W}$

$\lambda$ thermal conductivity, $\mathrm{W} \cdot \mathrm{m}^{-1} \cdot \mathrm{K}^{-1}$

\section{Subscripts}

ec

conduction

loss

received

hot

cold

initial

exterior

hot

\section{ANNEX}

Nomenclature and description of the commercially available insulating materials tested in the present study.

\begin{tabular}{ll}
\hline Sample number & Description \\
\hline 5 & Cellulose (recycle paper). \\
6 & Agglomerated polyurethane foam \\
& for wall insulation \\
7 & Rock-wool with density of $120 \mathrm{~kg} \cdot \mathrm{m}^{-3}$. \\
8 & Rock-wool covered with aluminum foil on \\
& one side \\
9 & Rock-wool (density, $150 \mathrm{~kg} \cdot \mathrm{m}^{-3}$ ) covered \\
& with a bitumen layer \\
\hline
\end{tabular}

